\section{Case Reports in Dermatology}

\title{
Lipofilling: A New Therapeutic Option for the Treatment of Lupus Panniculitis-Induced Atrophy
}

\author{
Laura Polivka $^{\mathrm{a}-\mathrm{c}}$ Marc Revol $^{\mathrm{a}, \mathrm{d}} \quad$ Maxime Battistella $^{\mathrm{a}, \mathrm{e}}$ \\ Hervé Bachelez ${ }^{a-c}$ \\ ${ }^{a}$ Paris Diderot-Sorbonne Paris Cité University, Paris, France; ${ }^{b}$ Department of Dermatology, \\ Saint Louis Hospital (AP-HP), Paris, France; 'INSERM Unit U1163, Institut Imagine, \\ Necker-Enfants Malades Hospital, Paris, France; ${ }^{d}$ Department of Plastic Surgery, \\ Saint Louis Hospital (AP-HP), Paris, France; ${ }^{e}$ Department of Pathology, Saint Louis \\ Hospital (AP-HP), Paris, France
}

\section{Keywords}

Lupus panniculitis · Lipofilling · Subcutaneous atrophy

\begin{abstract}
Lupus panniculitis is a rare manifestation of cutaneous lupus erythematosus, which may lead to major aesthetic sequelae with a severe impact on patients' quality of life. We report 2 cases supporting the short- and long-term efficacy and safety of lipofilling in the treatment of lupus panniculitis-induced atrophy. These observations pave the way for prospective, largerscale studies in patients with scarring lupus panniculitis, provided that the autoimmune pathogenic process is in complete, stable remission.

(C) 2016 The Author(s) Published by S. Karger AG, Basel
\end{abstract}

\section{Introduction}

Lupus panniculitis (LP) occurs in 3\% of the patients with cutaneous lupus erythematosus. It primarily affects subcutaneous tissues of the face and the proximal areas of the limbs, leading to residual atrophy [1]. LP may lead to major aesthetic sequelae that severely decrease quality of life (even during good long-term control of the autoimmune processes). 
Given the condition's low prevalence, treatment strategies in LP have not been extensively investigated. Hydroxychloroquine is generally considered the first-line treatment [2]. Correction of the aesthetic sequelae has rarely been investigated [3]. Only 1 case of lipofilling has been reported with a follow-up period limited to 12 months [4]. Here, we report on the strikingly good aesthetic outcomes, the long-term quality-of-life benefits, and the safety of lipofilling in 2 patients with LP-associated subcutaneous atrophy.

\section{Case Presentations}

Patient 1 (a 13-year-old boy) was referred for an ill-defined subcutaneous inflammatory nodule on the chin. A histopathological examination revealed a marked lymphocytic infiltrate (in superficial and deep tissues) with juxtafollicular involvement, prompting us to diagnose LP. There were no symptoms of systemic lupus erythematosus, and antinuclear antibodies were negative. Treatment with hydroxychloroquine for 18 months led to the complete disappearance of inflammatory signs but resulted in a cup-shaped lipoatrophy and facial asymmetry.

Patient 2 (a 32-year-old female) had been monitored over a 6-year period for systemic lupus erythematosus with positive antinuclear and anti-double-stranded DNA antibodies. During treatment with hydroxychloroquine, she presented with subcutaneous inflammatory lesions on the thighs and cheeks. A diagnosis of LP was confirmed by histopathological examination of a skin biopsy according to the criteria by Peter and Su [5]. The initial response to oral prednisone was good, but the patient became steroid dependent. A series of steroidsparing agents was then administered (including thalidomide, cyclophosphamide, and azathioprine). but all failed to induce stable remission. Four weekly intravenous infusions of rituximab $\left(375 \mathrm{mg} / \mathrm{m}^{2}\right)$, which were repeated 1 year later, led to complete remission, although atrophic scarring in malar areas persisted.

Following a request from both patients for correction of the aesthetic sequelae, we chose to use lipofilling. The abdominal liposuction procedure was performed using the Coleman technique [6]. Briefly, $10 \mathrm{~mL}$ of fat were collected via a 2-mm incision below the umbilicus. The tissue was centrifuged at 4,000 rpm for $3 \mathrm{~min}$. Next, fat cells were injected into the submental (Patient 1) or malar (Patient 2) areas using Coleman needles. The increase in the subcutaneous fat volume was immediately noticeable. There were no complications, and the positive aesthetic outcome was maintained 3 years after the procedure with no signs of the recurrence of LP (Fig. 1). The Dermatology Quality of Life Index decreased from 16 before lipofilling to 0 six months thereafter (Patient 1 ).

\section{Discussion}

Lipofilling is a well-established technique widely used in the rejuvenation of the aging face and in iatrogenic lipoatrophy $[7,8]$. This technique was also used successfully in some cases of linear scleroderma en coup de sabre [9]. Most of the common side effects of lipofilling are not severe. Nevertheless, it is critical for surgeons to have a firm knowledge of the vascular anatomy because some cases of irreversible blindness have been reported [10]. The good response of Patient 2 to rituximab suggests that the efficacy of rituximab as a salvage therapy for treatment-refractory LP should be assessed in prospective trials [11]. 
Along with a previous report, the 2 present cases suggest that lipofilling is effective for treating the atrophic scars that result from permanent alterations of the subcutaneous fatty tissue in LP. Moreover, the short- and long-term absence of any signs of lupus relapse or postsurgical complications is encouraging. The lipofilling procedure appears to be very safe when performed in patients with stable disease and thus paves the way for prospective, larger-scale studies in patients with completely controlled autoimmune disorder. These long-term, controlled trials will be able to accurately assess the benefit-risk ratio of lipofilling in LP and other immune-related subcutaneous scarring conditions.

\section{Statement of Ethics}

Both patients gave their informed consent to the publication of this article.

\section{Disclosure Statement}

The authors declare that no conflicts of interest exist.

\section{References}

1 Jarukitsopa S, Hoganson DD, Crowson CS, Sokumbi O, Davis MD, Michet CJ, et al: Epidemiology of systemic lupus erythematosus and cutaneous lupus in a predominantly white population in the United States. Arthritis Care Res (Hoboken) 2015;67:817-828.

-2 Braunstein I, Werth VP: Update on management of connective tissue panniculitides. Dermatol Ther 2012;25:173-182.

-3 Eastham AB, Liang CA, Femia AN, Lee TC, Vleugels RA, Merola JF: Lupus erythematosus panniculitis induced facial atrophy: effective treatment with poly-L-lactic acid and hyaluronic acid dermal fillers. J Am Acad Dermatol 2013;69:e260-e262.

-4 Valdatta L, Cherubino M, Tamborini F, Pellegatta I, Maggiulli F: A case of facial lipoatrophy secondary to lupus profundus managed with lipofilling technique. Case Rep Dermatol Med 2012;2012:720518. Peters MS, Su WP: Lupus erythematosus panniculitis. Med Clin North Am 1989;73:1113-1126. Coleman WP: Autologous fat transplantation. Plast Reconstr Surg 1991;88:736.

Mori A, Lo Russo G, Agostini T, Pattarino J, Vichi F, Dini M: Treatment of human immunodeficiency virus-associated facial lipoatrophy with lipofilling and submalar silicone implants. J Plast Reconstr Aesthetic Surg JPRAS 2006;59:1209-1216.

8 Dasiou-Plakida D: Fat injections for facial rejuvenation: 17 years experience in 1,720 patients. J Cosmet Dermatol 2003;2:119-125.

-9 Ibler KS, Gramkow C, Siemssen PA: Autologous fat transplantation for the treatment of linear scleroderma en coup de sabre. Skinmed 2015;13:74-76.

10 Beleznay K, Carruthers JDA, Humphrey S, Jones D: Avoiding and treating blindness from fillers: a review of the world literature. Dermatol Surg 2015;41:1097-1117.

11 Moreno-Suárez F, Pulpillo-Ruiz A: Rituximab for the treatment of lupus erythematosus panniculitis. Dermatol Ther 2013;26:415-418. 


\section{Case Reports in Dermatology}
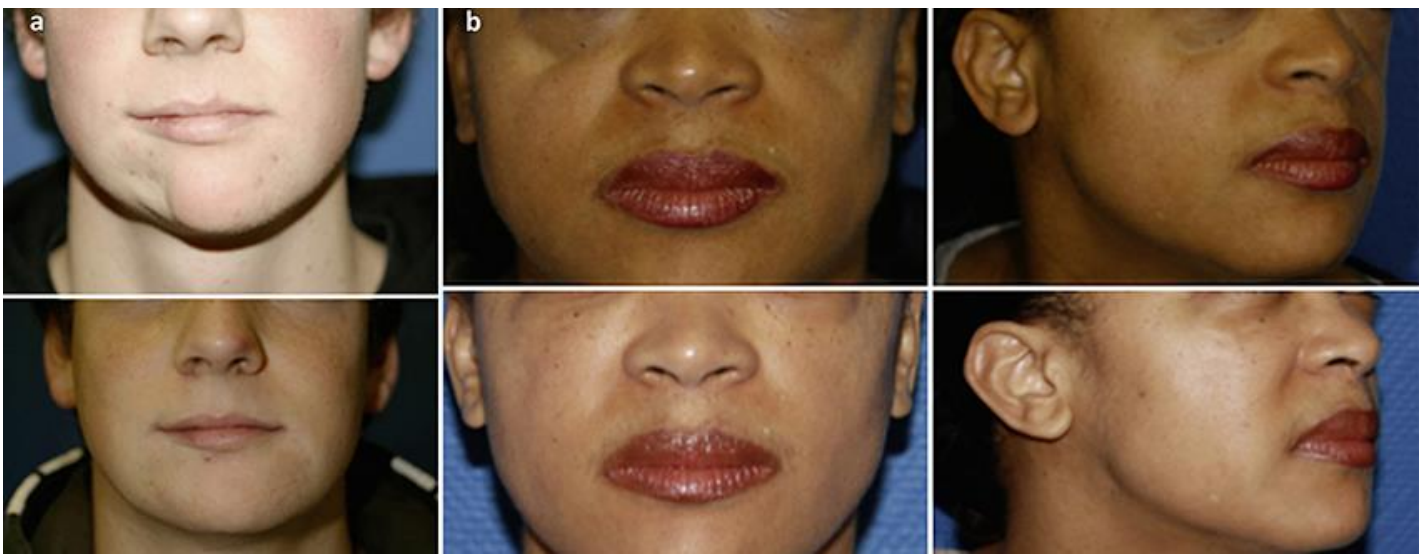

Fig. 1. Clinical findings of Patients 1 and 2. a The chin scar of Patient 1 on the day before surgery (upper panel) and 14 months afterwards (lower panel). $\mathbf{b}$ The atrophic scars under both eyes of Patient 2 on the day before the surgery (upper panels) and 3 years afterwards (lower panels). 